\title{
BACTERIAL PROFILE AND ANTIMICROBIAL SUSCEPTIBILITY PATTERN OF ISOLATES AMONG BURN PATIENTS AT YEKATIT 12 HOSPITAL BURN CENTER, ADDIS ABABA, ETHIOPIA
}

\author{
Tsegaye Sewunet ${ }^{1}$, Yohanes Demissie $^{2}$, Adane Mihret $^{3}$, Tamrat Abebe $^{3}$
}

\begin{abstract}
BACKGROUND: Infection is a common cause of morbidity and mortality in burn patients. Clinical diagnosis of bacteremia and/or sepsis in burn patients is difficult for a number of reasons. It could be symptomatic and/or asymptomatic as a result of immune deficiency secondary to thermal injury.

METHODS: A cross sectional study was conducted at Yekatit 12 Hospital Burn Center. Blood specimen and wound swab were collected from burn patients and were cultured by conventional method. Sensitivity/susceptibility pattern of the isolates was determined by disc diffusion method. Some of the risk factors of bacteremia like prior antibiotic use and total body surface area burn were also determined.

RESULTS: Fifty patients were enrolled in the study of whom 21(42\%) were found bacteremic. Five different bacteria were isolated from blood specimen. Coagulase negative Staphylococci, 9(42.8\%), S. aureus, 8(38.2\%), Bacillus spps, 2(9.52\%), K. pneumoniae, 1(4.8\%), and P. aeruginosa, 1(4.8\%), were frequent isolates. From wound swab, S. aureus, (34.04\%), and P. aeruginosa, (31.8\%), were predominant. Antimicrobial resistance was observed for Ampicillin, (77.4\%), Doxycycline, (74.0), Nalidixic acid, (70.5\%), Penicillin G, (68.2\%), and tetracycline, (67.5\%). Total body surface area of burn $\geq 15 \%$ was found as a risk factor for bacteremia.

CONCLUSION: Bacteremia was detected at a rate of $42 \%$ among burn patients. Frequent isolates were S. aureus, (34.04\%), and P. aeruginosa, (31.8\%). About $82.16 \%$ of the isolates showed multiple resistances. In light of our findings, regular antibiotic resistance test has to be done for each patient in order to select an appropriate antimicrobial agent.

KEYWORDS: Bacteraemia, Burn, Sepsis, Thermal injury
\end{abstract}

DOI: http://dx.doi.org/10.4314/ejhs.v23i3.3

\section{INTRODUCTION}

Burn wound infection is one of the most common causes of mortality and morbidity in burn patients (1). Major burn can be defined as any burn that requires intravenous resuscitation fluid or covers $10 \%$ of body surface area in children and $15 \%$ of the body surface area in adults; and/or also burn that involves the air way (2-6).

The frequency and gravity of septic complications in seriously ill burn patients impose the need for an accurate diagnostic and therapeutic approach. The microbiological monitoring of surface swabs and biopsies from burn wounds provide precise information as about the type of infection and the bacterial charge, but they give no indication of the depth of the infection (7-12). Microbiological investigation of superficial swabs should therefore be complemented with histological examination in order to detect possible presence of microorganism beneath the eschar. However, it is time-consuming and expensive, making it

\footnotetext{
${ }^{1}$ Department of Microbiology, Jimma University, Jimma, Ethiopia

${ }^{2}$ Department of Surgery, Addis Ababa University, Addis Ababa, Ethiopia

${ }^{3}$ Department of Microbiology, Immunology and Parasitology, Addis Ababa University, Ethiopia

Corresponding Author : Tsegaye Sewunet, Email : mbfj2004@yahoo.com
} 
impractical as a routine diagnostic technique; diagnosis of infection therefore relies on clinical parameters with the aid of blood and surface or tissue/biopsy cultures to identify the likely pathogen (13-17).

Infection in burn patient is a leading cause of morbidity and mortality and it continues to be the challenging concern; the importance of preventing infection has been recognized in organized burn care centers starting from its inception. These included strict asceptic techniques, use of sterile gloves and dressing materials, wearing masks for dressing changes and special separation of patients, using private rooms (14).

Clinical diagnosis of bacteremia and/or sepsis is difficult for a number of reasons. It could be symptomatic and/or may be asymptomatic as a result of immune deficiency secondary to thermal injury, malnutrition, anemia (reperfusion impaired), and damage to barriers (immunological) $(15,16)$. Periodical culturing and surveillance of potential microorganisms and their sensitivity/susceptibility pattern may alert early management and possible decrease in morbidity and mortality of burn patients from septicemia. The nature of microbial colonization of the wound, flora changes, and antimicrobial sensitivity profiles should be taken in to consideration in using empirical antimicrobial therapy for burn patients. This study was conducted to determine bacteremia, bacterial profile, and antimicrobial susceptibility pattern of bacterial isolates.

\section{MATERIALS AND METHODS}

A cross sectional study was conducted at Yekatit 12 Hospital Burn Center; Addis Ababa, Ethiopia from April to July 2010. Ten milliliters of blood specimen was collected from the fore arm after cleaning with $70 \%$ alcohol. Then, the needle used for collection was replaced by new sterile needle for dispensing into culture bottles. The blood specimens were inoculated into a trypton soya broth, and incubated at aerobic and anaerobic conditions (using anaerobic gas generating kits). The inoculated bottles were inspected daily for a sign of growth (hemolysis, turbidity, surface growth), and sub-cultured on blood agar (Oxoid) and MacConckey agar (Oxoid) when there was a sign of growth to isolate pure colony. Finally, the bottle with no growth after seven days was sub- cultured on blood agar and MacConckey in a similar way like bottles showing growth, and rejected if there was no growth after sub-culture. After isolation of the pure colony, further biochemical identifications were done by conventional methods.

Sufficient wound swabs were also collected. Wound swabs were inoculated on (blood agar, MacConckey, and Mannitol salt agar (Oxoid) and incubated for 24 hours. Each identified growth was characterized biochemically. Antimicrobial susceptibility patterns for each isolate were determined by disk diffusion (Kirby-Bauer technique) on Muller Hinton agar (Oxoid) for the commonly prescribed drugs at the Burn Center.

The bacterial isolates were tested against the following drugs commonly used at the Burn Center; Ampicillin $(10 \mu \mathrm{g})$, Amoxicillin $(30 \mu \mathrm{g})$,

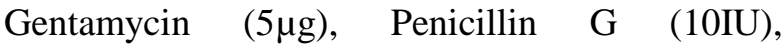
Methicillin $(5 \mu \mathrm{g})$, Amoxicillin/clavulinic $(20 \mu \mathrm{g})$, Sulphomethaxazole/trimethprime $(25 \mu \mathrm{g})$, Norfloxacin $(10 \mu \mathrm{g})$, Ciprofloxacin $(5 \mu \mathrm{g})$ and Nalidixic acid $(30 \mu \mathrm{g})$ (Oxoid). The data was analyzed by using SPSS version16 statistical software for descriptive statistics.

The specimen was collected after the study was approved by the Ethics Committee of Addis Ababa University-Institutional Review Board (AAU-IRB); and written consent was obtained from the study participants and from parents or legal guardians for study participants under the age of 18 .

Patients under critical condition, unconscious patients and patients who were not willing to participate in the study were not included in the study.

\section{RESULTS}

A total of 50 burn patients who either visited or were admitted to the Burn Center during the data collection period were included in the study. Both blood and wound swab samples were collected from all study subjects. Of the total study participants, females accounted for 20(40\%) and males accounted for 30 (60\%), whereas the age ranged from 7 years to 55years with the mean and median ages of 26.24 years and 24.5 years respectively.

The magnitude of bacteremia among burn patients at the center was 21(42\%). Five different bacterial species were isolated; Coagulase 
negative staphylococci and Staphylococcus aureus were most common. The distribution of these isolates ranged between Coagulase negative staphylococci, 9(42.8\%), S. aureus, 8(38.2\%), Bacillus spps 2(9.52\%), and both Klebsella pneumoniae and Pseudomonas aeruginosa, 2(4.8\%), (Table1).

Table 1: Magnitude and types of bacteria isolated from the blood culture of burn patients at the Yekatit12 Hospital Burn Unit, Addis Ababa, Ethiopia (July 2010).

\begin{tabular}{ll}
\hline Types of isolates & Number (\%) \\
\hline Coagulase negative staphylococci & $9(42.8)$ \\
Staphylococcus aureus & $8(38.2)$ \\
Bacillus spps & $2(9.52)$ \\
Pseudomonas aeruginosa & $1(4.8)$ \\
Klebsiella pneumoniae & $1(4.8)$ \\
Total & $\mathbf{2 1 ( 1 0 0 )}$ \\
\hline
\end{tabular}

Bacterial agents were isolated from 39 of 50 wound swabs. The total bacterial isolates were 47 and were identified as; S.aureus and $P$. aeruginosa were predominant isolates, 16(34.04\%), and $15(31.8 \%)$, respectively, followed by Coagulase negative Staphylococci 6(12.76\%), Proteus vulgaris 4(8.5\%), Proteus mirabilis 4(8.5\%), Klebsella pneumoniae 1(2.1\%), and Providencia spps $1(2.1 \%)$. Polymicrobial colonization of the wound was seen in 7/39 (17.94\%) wound samples (Table 2).

Table 2: Distribution and types of the bacterial isolates from the burn wound swab of burn patients at the Yekatit 12 Hospital Burn Center, Addis Ababa, Ethiopia (May- July 2010).

\begin{tabular}{ll}
\hline Types of isolates & $\begin{array}{l}\text { Number } \\
(\%)\end{array}$ \\
\hline Staphylococcus aureus & $16(34.04)$ \\
Pseudomonas aeruginosa & $15(31.8)$ \\
Coagulase negative Staphylococci & $6(12.76)$ \\
Proteus mirabilis & $4(8.5)$ \\
Proteus vulgaris & $4(8.5)$ \\
Klebsiella pneumoniae & $1(2.1)$ \\
Providencia spps & $1(2.1)$ \\
Total & $\mathbf{4 7 ( 1 0 0 )}$ \\
\hline
\end{tabular}

The total bacteria identified in this study both from blood and wound were 68 both at inpatient and outpatient care services. The distribution of these species varies with $S$. aureus 24(35.3\%), $P$. aeruginosa $16(23.5 \%)$, Coagulase negative Staphylococci 15(22.1\%), P. mirabilis 4(5.9\%), $P$. vulgaris 4(5.9\%), Bacillus species 2(2.9\%), $K$. pneumoniae 2(2.9\%) and Providencia spps $1(1.5 \%)$ (Table 3$)$.

Table 3: Distribution and magnitude of the bacterial isolates both from the burn wound swab and blood culture from burn patients at the Yekatit 12 Hospital Burn Center, Addis Ababa, Ethiopia (May- July, 2010).

\begin{tabular}{|c|c|}
\hline Types of isolates & Number (\%) \\
\hline S. aureus & $24(35.5)$ \\
\hline Pseudomonas aeruginosa & $16(23.5)$ \\
\hline $\begin{array}{l}\text { Coagulase } \\
\text { staphylococci }\end{array}$ & $15(22.1)$ \\
\hline Proteus mirabilis & $4(5.9)$ \\
\hline Proteus vulgaris & $4(5.9)$ \\
\hline Bacillus species & $2(2.9)$ \\
\hline Klebsiella pneumoniae & $2(2.9)$ \\
\hline Providencia spps & $1(1.5)$ \\
\hline Total & $68(100)$ \\
\hline
\end{tabular}

With regard to the antimicrobial susceptibility/sensitivity pattern, all the isolates were tested against the following commonly prescribed drugs at the center and widely used in the country: Norfloxacin, Augmentin, Nalidixic acid, Penicillin G, Gentamycin, Ceftriaxone, Doxycycline, Ampicillin, Methicillin, and Tetracycline. Among the tested drugs, Norfloxacin was not found to have resistance and shown intermediate resistance to one isolate only; Nalidixic acid (77.4\%), Ampicillin (76.1\%), Doxycycline $(74.1 \%)$, Penicillin G $(68.3 \%)$ and Tetracycline $(67.8 \%)$, had the highest resistance (Table 4).

Some of the possible risk factors for bacteremia like the total body surface area (TBSA) burn, prior antibiotic use, medical care service and area of residence were recorded. All the bacteremia cases, 21(100\%), were isolated from the 42 patients with the TBSA of $\geq 15 \%$; $38 / 42$ (90.4\%) patients who were positive for wound swab culture were also from the group of patients with $\mathrm{TBSA} \geq 15 \%$. Other factors like 
prior systemic antibiotics use showed relatively decreased proportion of bacteremia 5/14(35.7 \%) as compared to non users16/36 (44.4\%). The proportion of wound swab isolates both among the groups of patients who had used antibiotic before coming to the center and those who had not used remained more or less similar 11/14 (78.5\%) and 28/36 (77.7\%), respectively.

Table 4: Antimicrobial sensitivity/susceptibility pattern of the bacterial isolates both from the burn wound swab and blood culture from burn patients at the Yekatit 12 Hospital Burn Center, Addis Ababa, Ethiopia (May-July, 2010).

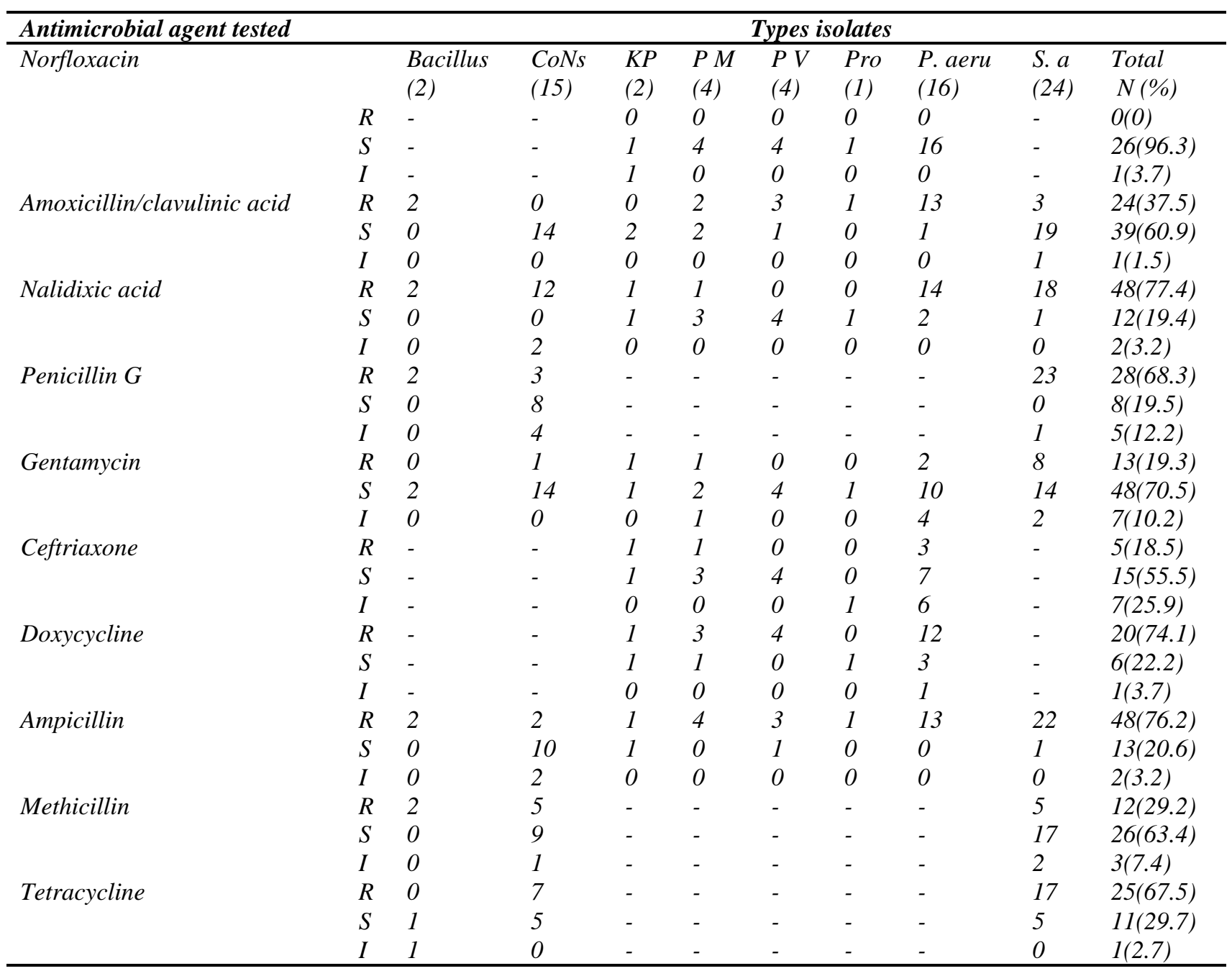

$\mathrm{R}=$ resistance; $\mathrm{S}=$ sensitive; $\mathrm{I}=$ intermediate, - = not tested $1 ; \mathrm{PM}=$ Proteus mirabilis, $\mathrm{PV}=$ Proteus vulgaris, $\mathrm{S} . \mathrm{a}=$ S. Aureus, $\mathrm{KP}=$ Klebsiella pneumoniae, $\mathrm{P}$ aeru $=$ Pseudomonas aeruginosa

\section{DISCUSSION}

The burn wound management and critical care medicine has accelerated a lot and is still developing with new generation tools being innovated. In burn patient care, bacteremia and /or septicemia is the major problem in burn patients. This study was conducted to determine the bacteriological profile and magnitude of bacteremia and has demonstrated $42 \%$ bacteremia. Bacterial isolates identified were Coagulase negative staphylococci (42.8\%), S. aureus (38.2\%) Bacillus spps (9.5\%), P. aeruginosa (4.8\%), and $K$. pneumoniae $(4.8 \%)$; these isolates were similar to bacterial isolates identified at other different burn centers $(6,11,12)$. 
Current definitions of sepsis and infection have many criteria (fever, tachycardia, tachypenia, and leukocytosis) that are routinely found in patients with extensive burns, making the current definition less applicable to the burn population $(20,21)$. This study has come up with bacteremia without having significant symptoms of sepsis according to the definition; which is still the terminal risk of sepsis and septic complications. Yet, it may seem clinically non-indicative but later when the bacterial number in the circulation is maintained at higher load, there could be an abrupt onset of the clinical sepsis that may accelerate to septic shock with poor prognosis. Delayed clinical symptoms of sepsis in the presence of bacteremia might be because of immunosuppression secondary to thermal injury. As another study (22) indicated temperature, white blood cell count, neutrophil percentage, or changes in these values were not clinically reliable in predicting blood stream infections. Further work is needed to identify the alternative clinical parameters which should prompt blood culture evaluation among the burn patient population.

Although the frequency of isolation is different from one burn center to the other, the bacteria profile remains similar. Generally, from both blood and wound swab, 68 bacterial isolates were isolated and characterized; these include: $S$. aureus (35.5\%), P. aeruginosa (23.5\%), Coagulase negative staphylococci, (22.1\%), Proteus vulgaris and Proteus mirabilis (5.9\%) each, Bacillus spps (2.9\%), Klebsella pneumonia, (2.9\%) to Providencia spps (1.5\%). When compared to one of the study (23) done at a tertiary care hospital in India, the isolates were all aerobic bacteria in a similar manner and predominantly $S$ aureus and $P$. aeruginosa accounting for $75 \%$ and they were multidrug resistant.

On the other hand, isolates from wound swabs were analyzed separately and hence the most common isolates from the wound swab include $S$. aureus (34.04\%), followed by $P$. aeruginosa (31.5\%), Coagulase negative staphylococci (12.76\%), Proteus mirabilis $(8.5 \%)$, Proteus vulgaris $(8.5 \%), K$. pneumoniae $(2.1 \%)$, and Providencia spps $(2.1 \%)$. Although a number of studies have been conducted on burn wound infection and bacterial profile, nearly all of them are retrospective studies which made comparison of findings of this study to those findings difficult; however, these studies remain optional for comparison. Comparison of bacterial isolates with other studies may also be difficult because of geographical variations, drug policies, infection control policies and the like $(23,24)$. Furthermore, particularly the resistant strains vary even between different intensive care units of the same institution (1).

The bacterial isolates in this study are more or less similar to the bacterial profile identified at Ain Shams University Hospital, Cairo (9). There is no anaerobic bacterium identified in this study which is also similar to the above mentioned study. Other studies $(23,25)$ have also indicated that anaerobic infections are rare. Rate of MRSA (7.3\%) is low as compared to a study (7) from India has shown that the dominant cause of wound infection is MRSA (71\%).

Of the drugs tested against these isolates, all the isolates were sensitive to Norfloxacin, except one organism $K$. pneumoniae which is intermediate. This study has shown a very high incidence of resistance to the drugs like Nalidixic acid (77.4\%), Ampicillin (76.2\%), Doxycycline (74.1\%), and Penicillin G (68.3\%), Tetracycline (67.8\%), Augumentin (37.5\%), Methicillin (29.5\%), Gentamycin (19.1\%), and Ceftriaxone $(18.5 \%)$ to the isolates identified at the burn center. Moreover, around $82.3 \%$ of the isolates were resistant to two or more of the drugs tested, demonstrating multiple drug resistance except for Norfloxacin.

Another serious problem at the critical care units is MRSA which is increasing from time to time and causes increased cost (26). The MRSA incidence in the present study is $(7.3 \%)$, although it is lower compared to a study in India (71\%) (7), yet it is a critical problem in developing countries where drug spectrum is limited. This significant difference might be the result of the difference in epidemiological parameters of MRSA and might also be because of drug policy. In addition, the period of study and sample size might also cause the difference. The increase in Coagulase negative staphylococci isolates was significant because it might be associated with Methicillin resistant Coagulase negative staphylococci strains. Coagulase negative staphylococci is recognized to be equally pathogenic as $S$. aureus in immune suppressed subjects such as thermal injuries and 
often deliver its genetically resistant codes to previously liable S.aureus which subsequently turns in to MRSA (24). This might be why MRSA and Methicillin resistant Coagulase negative staphylococci increase concomitantly. Another study has determined that death related to bacterial blood stream infection is commonly because of $S$. aureus early in the hospital course (27).

The higher incidence of resistant isolates could be because of the inappropriate use of antibiotics. To the knowledge of investigators, there are no antibiotic use policies and infection control units in any of the care centers or health institutions in the country. Despite the fact that $S$. aureus and other common bacterial agents are similar at different burn centers; the antimicrobial sensitivity pattern cannot be compared between these centers because of difference in different prescription patterns and may be because there is no standardized prescription and management scheme common to all. However, the antibiotic treatment should be changed in accordance with the observed antibiotic susceptibility pattern in case of positive blood cultures and signs of sepsis. Because of the insufficient supply of drugs, the antimicrobial treatment choice and changes has to be made to the wise utilization of what is available at the time.

The total body surface area (TBSA) of the burn; a TBSA of $\geq 15 \%$ cut off value as a major burn was considered a risk factor for bacteremia. All cases of bacteremia (100\%) in this study were isolated from patients having TBSA of burn $\geq 15 \%$. The higher the total body surface area damaged by the thermal assault the higher the potential for the bacteria to colonize and proliferate increasing the wound thickness and depth making way to the blood stream involvement.

Bacteremia was found among the burn patients at a magnitude of $42 \%$ at the burn center. The most common isolates at this center were $S$. aureus and $P$. aeruginosa. Almost all isolates were multiple drug resistant isolates; $82 \%$ of the isolates showed resistance to at least two or more of the drugs tested. Tetracycline, Ampicillin and Nalidixic acid were the drugs to which higher percentage of resistance has been seen among the tested drugs whereas Norfloxacin was the only drug to which all isolates tested were found sensitive. Risk factors associated with bacteremia were TBSA of burn $\geq 15 \%$, area of residence; being from rural area has showed higher proportion of wound infection isolates. Prior systemic antibiotic use was also found to decrease risk of bacteremia but has no effect on wound infection.

Bacteremia goes undetectable at a rate of $42 \%$ among burn patients at Yekatit-12 Hospital Burn Unit. The most common bacterial isolates were Coagulase negative staphylococci and Staphylococcus aureus. The distribution of these isolates ranges between Coagulase negative staphylococci, 9(42.8\%), S. aureus, 8(38.2\%), Bacillus spps, 2(9.52\%), and both Klebsella pneumoniae and Pseudomonas aeruginosa, $2(4.8 \%)$. Total body surface area of burn (TBS) greater than $15 \%$ was found to be the risk factor for bacteremia.

Bacterial isolates identified from wound swab were 47 ; S.aureus and $P$. aeruginosa were predominant isolates, 16(34.04\%), and 15(31.8\%), respectively, followed by Coagulase negative Staphylococci 6(12.76\%), P. vulgaris 4(8.5\%), P. mirabilis $48.5 \%), K$. pneumoniae $1(2.1 \%)$, and Providencia spps 1(2.1\%). Polymicrobial colonization of the wound was seen from $7 / 39$ (17.94\%) wound samples.

Among the tested drugs, Norfloxacin was not found to have resistance and shown intermediate only for one isolate. Nalidixic acid, (77.4\%), Ampicillin, (76.1\%), Doxycycline, (74.1\%), Penicillin G, (68.3\%), and Tetracycline, (67.8\%), have the highest resistance. And $82.16 \%$ of the isolates in this study showed multiple resistances (at least two or more of antibiotics tested).

For burn patient care, bacteriological blood culture, wound swab culture and antimicrobial susceptibility pattern of isolates should be done for each patient under ideal conditions. However, this is not possible for resource limited countries, and hence, periodic surveillance of the burn unit isolates should be done to know commonly circulating isolates. This helps clinicians to consider appropriate antimicrobial agents in hand when empirical treatment is a mandatory option in critical situations, which is a common practice in burn units. 


\section{ACKNOWLEDGEMENTS}

We acknowledge Jimma University for some material support. We have special thanks to Addis Ababa University for the financial support. We are also thankful to Mr. Ayelign Derbie for unreserved technical assistance.

\section{REFERENCES}

1. Sedat Y, Tarik Z, Nurkan T, Targut N, Yusuf Z, Gokhan M, Mehmet H. Bacteriological profile and antibiotic resistance: Journal of burn wound and rehabilitation. 2005; 26(6):488-

2. Warrick A. Ames. Management of the major burn, update in anesthesia. 1999; 10 (10)

3. Clinton M, Duane R. Burn wound infection: follow up, infectious disease fellowships 2008. http://www.emedicine.mediscape.com

4. Clinton M, Duane R. Burn wound infections, health education consortium 2008 http://www.emedicine.mediscape.com

5. Forjouh S.N. Burns in low and middle income countries. Burns 2006; 32 (12), 529-537.

6. Al Akyaleh A.T, Invasive burn wound infection. Ann Burns Fire disasters. 1999; 12 (4): $1-5$.

7. Mago V. Burn wound septicemia, analysis of burn wound infections burn ward. Journal of Burn care and Research. 2009; 30(3):540.

8. Kehinde A.O, Ademola S.A, Okesola A.O, Oluwatosin O.M, Bakare R.A. Pattern of bacterial pathogens in burn wound infection, in IBADAN, Nigeria, Ann Burns Fire Disasters. 2004; 17(1):12-15.

9. Nasser S, Mabrouk A, Maher A. Colonization of burn wound infection Ain Shams University, burn unit; Burns 2003; 29(3):229.

10. Sanjay D, Rakesh S, Kailash S, Bhavani R. Microbiological profile of chronic burn wounds among patients admitted to the burn unit; JK science 2007; 9 (4):182-185.

11. Herjinder K, Jyothi B, Anup R, Savinder R, Vijay G. Bacterial profile of blood and burn wound infections in burn patients, proceeding of national symposium on Tribal Health, 2008.

12. Alireza E, Enayat K. Analysis of bacterial infections in burn patients at Talenghani burn
Hospital in Ahvaz. Iranian journal of clinical infectious diseases, 2007; 2(1):9-12.

13. Neely A, Gardner J, Durkee P, et al. Are topical antimicrobials effective against bacteria that are highly resistant to systemic antibiotics, Journal of Burn care and Research 2009; 30(1):19-29.

14. Sharma B.R, Virendar P, Sumedha B and Neha G. Septicemia the principal killer of burn patients, American journal of infectious diseases. 2005; 1(3): 132-138.

15. Ulku A, Serpil A, Mufide N, Fehimi A, Ayten A. The time related changes of anti microbial resistance patterns and predominant bacterial profiles of burn wounds and body flora of burned patients, Journal of the international society of burn injuries. 2004; 30(7):660-664.

16. Di Lonardo A, Ferrante M., Maggio G., Bucaria V., Del Zotti M., Brienza E. Histological assessment of the level of burn wound infection: diagnostic and therapeutic strategies. Ann. Medit. Burns Club.1993; 6(3):433-5.

17. Mark A, Carolyn H. ABC of Burns, intensive care management. BMJ 2004; 329 (12).

18. Ressner A., Murray K., Griffith E., Rosnake S., Hospenthal R., Wolf E. Outcomes of bacteremia in burn patients involved in combat operation overseas. Journal of American college of Surgeons. 2008; 26(3):439-444.

19. Deirdre C, Sameer E, Owen R, Brent W. Burn wounds and septicemia, clinical microbiology reviews. 2006; 19 (2): 403- 434.

20. Sjoberg T., S. Mzezewa, K.Jonsson, V.Robertson, L.Salemark. Comparison of surface swab cultures and quantitative tissue biopsy cultures to predict sepsis in burn patients: a prospective study. J Burn Care Rehabil. 2003; 24: 365-370.

21. David G, Jeffrey R, James $\mathrm{H}$, et al. The American burn association consensus conference on burn sepsis and infection in Burns. J Burn Care Res 2007; 28:776-790.

22. Clinton M, Roselle M, David R. et al. Evaluation of white blood cells count, neutrophil percentage, and elevated temperatures as predictors of blood stream infections in burn patients. Arch. Surg. 2007; 142(7):639-642. 
23. Anuradha R, K. P. Singh, Vijay K, Rishi S, R.K. Singh. Antibacterial resistance pattern of aerobic bacteria isolates of burn patients in tertiary care hospital. Biomedical research. 2008; 19(1):5-8.

24. Merlin G, Reinhard Z, Alexander E. H, Andreas G, Mehimet A, Pietro G. Changes in bacterial isolates from burn wounds and their antibiograms: a 20 year study (1986-2005). Burns 2009; 553-560.

25. Agnihorti N., V. Gupta, R.M. Joshi. Aerobic bacterial isolates from burn wound infections and their antibiograms. Burns 2004; 30: 241243.

26. Webennmeyer L, Appelgate D, Williams I, et al. Effectiveness of universal screening for vancomycin resistant enterococci and methicillin resistant staphylococcus aureus on admission to a burn. Journal of Burn care and research 2009; 30(4):648-656.

27. Laurie $\mathrm{C}$, Brian $\mathrm{K}$, Clinton $\mathrm{K}$ et al. Contribution of bacterial and viral infections to attributable mortality in patients with severe burns; an autopsy series. Burns. 2010; 36 (6): 773-779. 